Rytel-Schwarz Danuta / Jurasz Alina / Cirko Lesław / Engel Ulrich, 2018, Deutsch-polnische kontrastive Grammatik. Band 2: der Satz, 2., neu bearbeitete und ergänzte Auflage, Hildesheim. Wahrig. Deutsches Wörterbuch, 2011, München.

\title{
Magdalena Zofia Feret (2014), Die Nominalphrase im Deutschen aus der Perspektive der generativen DP-Modelle und der kognitiven Gram- matik. Ein Versuch der Bewertung ihrer Untersuchungsmethodologien, Kielce: Wydawnictwo Uniwersytetu Jana Kochanowskiego, 298 S., ISBN 978-83-7133-612-6.
}

DOI: $10.19195 / 0137-1169.38 .11$

Das Buch von Magdalena Zofia Feret unter dem Titel „Die Nominalphrase im Deutschen aus der Perspektive der generativen DP-Modelle und der kognitiven Grammatik. Ein Versuch der Bewertung ihrer Untersuchungsmethodologien“ beschäftigt sich mit den Untersuchungsmethodologien von ausgewählten generativen Modellen (Abney 1987, Chomsky 1981, 1986, 1995, 2000) und der kognitiven Grammatik von Langacker $(1987,1991)$. Das Hauptinteresse der Sprachforscherin gilt der deutschen Nominalphrase, die einerseits im Lichte von Theorien der generativen Grammatik, andererseits vom kognitiven Modell betrachtet wird. Während die erste Vorgehensweise die syntaktischen Prozesse fokussiert, welche den Mechanismen der Nominalphrasengenerierung zu Grunde liegen, und somit deren präzise und systematische Beschreibung ermöglicht, richtet die kognitive Grammatik ihr Hauptaugenmerk auf die konzeptuell-semantischen Prozesse, die mit der Beschreibung der Bedeutung von Nominalphraseneinheiten in Zusammenhang stehen. Die Publikation ist somit als Brückenschlag zwischen den beiden sprachwissenschaftlichen Ansätzen zu betrachten. Die beiden Theorien vertreten eine mentalistische Sprachauffassung, auch wenn sie sich in der Art und Weise der Wahrnehmung der mentalen Wirklichkeit deutlich voneinander unterscheiden. Die Zielsetzung, die Phänomene im nominalen Bereich des Deutschen von zwei unterschiedlichen Perspektiven aus zu analysieren, scheint ein garkühnes Unterfangen zu sein, weil sie eine detaillierte, äußerst sorgfältige und sehr subtile Auseinandersetzung mit den oben genannten Fragestellungen erfordert, was die Buchautorin vortrefflich zu meistern vermochte. Das Innovative der Monographie besteht darin, durch die Nebeneinanderstellung zweier gegensätzlicher Perspektiven ein facettenreiches Bild von den aufgegriffenen Phänomenen geliefert zu haben. Die methodologischen Überlegungen sorgten überdies für die Entstehung einer sorgfältigen und ideenreichen Arbeit, zu der man nur gratulieren kann.

Die Gliederung des Inhalts ist im Buch transparent und durchdacht: Außer dem Kapitel mit der Einleitung in die untersuchte Problematik, dem Resümee und dem umfangreichen Literaturverzeichnis wurde das Buch in zwei Hauptteile gegliedert. Während der erste Teil der Syntax der Nominalphrase in generativer Sicht sowie der Semantik aus der kognitiven Perspektive gewidmet ist, wird die Bewertung der Untersuchungsmethodologien der generativen Modelle und des kognitiven Modells im weiteren Teil aufgegriffen. 
An dieser Stelle werden Definitionen des Begriffs Nominalphrase nach unterschiedlichen Auffassungen dargestellt, wobei folgende linguistische Modelle berücksichtigt werden: Heyse (1844), Bloomfield (1926), Wells (1947), Harris (1951), Fries (1952), Hockett (1958), Tesnière (1953, 1959) und Chomsky $(1956,1957,1965)$. Im Kapitel, das sich mit der Syntax der Nominalphrase beschäftigt, werden anhand der von der Autorin gesammelten und bearbeiteten Korpusdaten zahlreiche Beispiele von Nominalphrasen im Deutschen aus der Perspektive der generativen DP-Modelle analysiert. In dem angesprochenen Kapitel unternimmt die Autorin den gelungenen Versuch, die DP-Hypothese von Abney (1987) auf die Beschreibung der Nominalphrase im Deutschen anzuwenden. Parallel dazu werden ausgewählte Lösungen von deutschsprachigen Autoren wie Heider $(1988,1992)$, Bhatt (1990), Olsen (1991), Vater (1991, 1993, 1996, 2000, 2005) u.a. dargestellt und bewertet werden. Das der kognitiven Theorie gewidmete Kapitel stellt wiederum die Fragestellung der Nominalphrase im Kontext der Semantik dar, wobei hier der Hauptgedanke von Langacker $(1987,1991)$ sichtbar ist. Bei der Analyse der Beispiele aus dem von der Autorin bearbeiteten Korpus werden unterschiedliche Bedeutungsvarianten berücksichtigt und die Bedeutung der einzelnen Bestandteile von Nominalphrasen im Deutschen beschrieben.

Das u.E. relevanteste Kapitel des Buches enthält die Bewertung der Untersuchungsmethodologien der in der Arbeit angesprochenen linguistischen Modelle, deren theoretische Grundlage Überlegungen sowohl von Linguisten wie Chomsky (1965/83, 1982), Kardela (1992) und Bobrowski (1993) als auch von Methodologen (Popper 1935/2005, Lakatos 1978 und Więckowski 2000) bilden. Die interessantesten Schlussfolgerungen betreffen hierbei die Falsifizierbarkeit der diskutierten linguistischen Modelle und deren Auffassung als Forschungsprogramme im Sinne von Lakatos (1978).

Ohne Zweifel lässt sich behaupten, dass sich die Autorin eine schwierige Frage metalinguistischer Natur gestellt hat. Zweifellos verfügt sie über umfangreiche Kenntnisse im Bereich der linguistisch-methodologischen Fachliteratur und Theorien sowie über die Fähigkeit, die Lösungen bezüglich linguistischer Konzeptionen vorauszusehen. Zweifelsohne sieht man hier deutlich den roten Faden sowie eine konsequente Ausführung und eine ausführliche Argumentation, was das Buch kohärent macht. Darüber hinaus wird das Theoretische durch eine gründliche Analyse begleitet, die große Anerkennung finden soll, wobei die sprachliche und methodologische Präzision der Forscherin bemerkenswert sind.

Während der Lektüre des Buches bekommen wir einen wesentlichen Einblick in die Komplexität der beschriebenen Phänomene und können dies als Schritt in Richtung der Präzisierung der syntaktischen sowie semantischen Regeln im universellen Aspekt betrachten. Somit kann das Buch als eine Pionierarbeit betrachtet werden.

Die Veröffentlichung wurde sehr gut in der aktuellsten Literatur lokalisiert, wobei auch die älteren klassischen Literaturpositionen begründet berücksichtigt wurden. Dabei könnte man der Sprachforscherin vielleicht empfehlen mehr analytische Arbeiten zu diesem Thema einzusetzen, aber diese Bemerkung hat lediglich polemischen Charakter und will die Leistung der Autorin keinesfalls schmälern.

Piotr A. Owsiński

ORCID: 0000-0001-7862-3345

Uniwersytet Jagielloński, Kraków, Polen

Studia Linguistica 38, 2019

(C) for this edition by CNS 